\title{
Femtosecond Z-scan measurements of nonlinear refraction in nonlinear optical crystals
}

\author{
H.P. Li ${ }^{\text {a,* }}$, C.H. Kam ${ }^{\text {a }}$, Y.L. Lam ${ }^{\text {a }}$, W. Ji ${ }^{b}$ \\ a Photonics Laboratory, School of Electrical and Electronic Engineering, Nanyang Technological University, Nanyang Avenue, \\ Singapore 639798, Singapore \\ ${ }^{\mathrm{b}}$ Department of Physics, National University of Singapore, Lower Kent Ridge Road, Singapore 119260, Singapore
}

Received 1 June 2000; accepted 26 June 2000

\begin{abstract}
We report an investigation of third-order optical nonlinearities in several nonlinear optical crystals using the Z-scan technique with femtosecond laser pulses at $780 \mathrm{~nm}$ wavelength. The crystals studied include $\mathrm{LiNbO}_{3}: \mathrm{MgO}, \mathrm{KTiOAsO}_{4}$, $\mathrm{KTiOPO}_{4}, \beta-\mathrm{BaB}_{2} \mathrm{O}_{4}$ and $\mathrm{LiB}_{3} \mathrm{O}_{5}$, which are extensively used for ultrashort-pulse second-harmonic generation and optical parametric oscillation. The nonlinear refractive index $n_{2}$ in these crystals has been determined to be in the range from $10^{-16}$ to $10^{-15} \mathrm{~cm}^{2} / \mathrm{W}$. No two-photon absorption has been observed. The experimental results are compared with the two-band model for the bound electronic Kerr nonlinearity. It is shown that the measured $n_{2}$ values in $\beta-\mathrm{BaB}_{2} \mathrm{O}_{4}$ and $\mathrm{LiB}_{3} \mathrm{O}_{5}$ are one order of magnitude smaller than those of $\mathrm{LiNbO}_{3}: \mathrm{MgO}, \mathrm{KTiOAsO}_{4}, \mathrm{KTiOPO}_{4}$, which is in agreement with the theoretical prediction. (C) 2001 Elsevier Science B.V. All rights reserved.
\end{abstract}

PACS: $42.65 ; 42.70 ; 42.10$

Keywords: Nonlinear refraction; Nonlinear optical crystals; Z-scan technique

\section{Introduction}

$\mathrm{LiNbO}_{3}: \mathrm{MgO}$ (LN:Mg), $\mathrm{KTiOAsO}_{4}$ (KTA), $\mathrm{KTiOPO}_{4}(\mathrm{KTP}), \beta-\mathrm{BaB}_{2} \mathrm{O}_{4}(\mathrm{BBO})$ and $\mathrm{LiB}_{3} \mathrm{O}_{5}$ (LBO) are important nonlinear optical (NLO) crystals that have been extensively used in the applications of second-harmonic generation (SHG) and optical parametric oscillation (OPO). Nonlinear refraction of the crystals is of interest for these applications in the femtosecond time domain. The nonlinear refractive index, $n_{2}$, plays

\footnotetext{
${ }^{*}$ Corresponding author. Fax: +65-791-2687.

E-mail address: ps2682748e@ntu.edu.sg (H.P. Li).
}

an important role in the spatial and temporal pulse evolution in many $\chi^{(2)}$ mixing experiments involving ultrashort or high-energy optical pulses. Accurate knowledge of $n_{2}$ is of direct relevance in assessing spectral and temporal pulse broadening due to self-phase-modulation, chirp reversal and self-compression, the minimum pulse width attainable in ultrashort-pulse SHG and OPO devices $[1,2]$, and optical damage induced by self-focusing. It can also be used for the control of ultrashortpulse distortion effects through the optimized design of pulse shaping and nonlinear phase compensation schemes [3].

As a simple yet sensitive single-beam method, the Z-scan technique [4] has been developed for 
the measurement of third-order optical nonlinearities, particularly nonlinear refraction and nonlinear absorption. It is capable of providing information not only on the magnitude but also on the sign of the third-order susceptibility of a material. It can also distinguish between the real and imaginary parts of the third-order susceptibility $\left(\operatorname{Re} \chi^{(3)}\right.$ and $\left.\operatorname{Im} \chi^{(3)}\right)$, which can result from two-photon absorption (TPA) and optical Kerr effect, respectively. With the Z-scan technique, DeSalvo et al. have measured the TPA coefficient $(\beta)$ in both KTP and LN at $532 \mathrm{~nm}$, and the Kerr nonlinearity $\left(n_{2}\right)$ in KTP, LN and $\mathrm{BBO}$ at the four harmonics of fundamental $1064 \mathrm{~nm}$ wavelength $[5,6]$. By employing the same method, we have measured third-order optical nonlinearities of these NLO crystals and optical damage thresholds induced by self-focusing with $532 \mathrm{~nm}$ ps laser pulses [7]. To our knowledge, most of Zscan measurements on nonlinear absorption and refraction of NLO crystals were performed with picosecond pulses.

In this paper, we report an experimental investigation of refractive nonlinearity of important NLO crystals including $\mathrm{LN}: \mathrm{Mg}$, KTA, KTP, $\mathrm{BBO}$, and LBO. The investigation has been carried out using the Z-scan technique with 150 fs laser pulses at $780 \mathrm{~nm}$ wavelength. The microscopic origin of the measured Kerr nonlinearity is compared with a theoretical model based on bound electronic effects. It has been found that the measured $n_{2}$ values in $\mathrm{BBO}$ and $\mathrm{LBO}$ are one order of magnitude smaller than those of LN:Mg, KTA and KTP, which is consistent with the theoretical predication.

\section{Experimental}

In our Z-scan experiment, the laser pulses at $780 \mathrm{~nm}$ wavelength were delivered by a modelocked Ti:sapphire laser operating at a repetition rate of $76 \mathrm{MHz}$. The FWHM pulse duration was 150 fs. The spatial profile of the laser beam was nearly a Gaussian distribution after a spatial filter. The minimum beam waist $\omega_{0}$ of the focused laser beam was measured to be $13 \mu \mathrm{m}$. The linearly polarized pulses were divided by a beam-splitter into two parts: the reflected one used as a reference to represent the incident light power; and the transmitted one was focused through the sample. Both the beams were recorded by two power probes (Newport 818 SL) simultaneously, and measured by a dual channel power meter (Newport 2832-C) which transferred the digitized signals to a computer. The sample was mounted on a computer-controlled translation stage that moved the sample along the $z$-axis with respect to the focus of the lens. When measuring the nonlinear refraction, a pinhole was placed in front of the transmission detector. As a reference, we performed a Z-scan on a sample of polycrystalline $\mathrm{ZnSe}$, and obtained $n_{2}=2.3 \times 10^{-14} \mathrm{~cm}^{2} /$ $\mathrm{W}\left(1.0 \times 10^{-11} \mathrm{esu}\right)$ and $\beta=3.8 \times 10^{-9} \mathrm{~cm} / \mathrm{W}$, which are in good agreement with the published results [8].

The samples employed in this study were commercially supplied including LN:Mg (6 $\mathrm{mol} \%$ $\mathrm{MgO}$-doped $\mathrm{LiNbO}_{3}$ ), KTA, KTP, BBO, and LBO crystals cut perpendicular to the $z$-axis of the crystals. The thickness, $L$, of the samples is listed in Table 1. It should be pointed out that these

Table 1

Measured $n_{2}$-values in the $z$-cut NLO crystals with femtosecond $780 \mathrm{~nm}$ laser pulses polarized along [1 000$]$ and [0 10$]$ directions, respectively ${ }^{\mathrm{a}}$

\begin{tabular}{llcllll}
\hline Crystal & $L(\mathrm{~mm})$ & $L_{\mathrm{D}}(\mathrm{mm})$ & $E_{\mathrm{g}}(\mathrm{eV})$ & \multicolumn{2}{l}{$n_{2}($ Exp. $)\left(10^{-15} \mathrm{~cm}^{2} / \mathrm{W}\right)$} & $n_{2}($ theory $)\left(10^{-15} \mathrm{~cm}^{2} / \mathrm{W}\right)$ \\
\cline { 5 - 6 } & & & & {$[100]$} & {$[010]$} & \\
LN:Mg & 2 & 7.1 & 4.0 & $2.0 \pm 0.3$ & $2.0 \pm 0.3$ & 3.3 \\
KTA & 5 & 14 & 3.6 & $1.7 \pm 0.3$ & $1.7 \pm 0.3$ & 8.8 \\
KTP & 2 & 25 & 3.6 & $1.2 \pm 0.16$ & $0.94 \pm 0.16$ & 9.0 \\
BBO & 5 & 40 & 7.0 & $0.40 \pm 0.05$ & $0.32 \pm 0.05$ & 0.44 \\
LBO & 5 & 107 & 7.5 & $0.26 \pm 0.03$ & $0.19 \pm 0.03$ & 0.32 \\
\hline
\end{tabular}

\footnotetext{
${ }^{\text {a }}$ The theoretical $n_{2}$ values calculated according to Eq. (4) are also given.
} 
samples with 2- and 5-mm thickness did not satisfy the "thin sample" condition $L<n_{0} z_{\mathrm{R}}$ [4] in our Zscan experiments, where $n_{0}$ is the linear index of refraction of the crystal, and $z_{\mathrm{R}}=\pi \omega_{0}^{2} / \lambda$ the Rayleigh confocal parameter of the Gaussian beam. The UV-visible transmission spectra of the samples were measured using a Hitachi U-3040 spectrophotometer. From the cut-off energies in the linear transmission spectra, the band-gap energies $E_{\mathrm{g}}$ of the crystals were estimated as shown in Table 1.

Open- and closed-aperture Z-scan measurements were performed for the $z$-cut crystals at room temperature with the laser polarization along [ $\left.\begin{array}{lll}1 & 0 & 0\end{array}\right]$ and $\left[\begin{array}{lll}0 & 1 & 0\end{array}\right]$ directions of the crystals, respectively. It is noted that the second-order nonlinear optical susceptibility $\chi^{(2)}$ may manifest itself in the Z-scan through the $\chi^{(2)}: \chi^{(2)}$ cascading effect [9]. The effective $n_{2}$ arising from the cascading effect can be dominant in the near-phase-matched SHG processes. Our Z-scan measurements were performed with the 780-nm laser beam propagating along the $z$-axis of the crystals. Hence, the cascaded process was ignored due to the laser propagation direction far away from the phasematching direction. Our experimental error was about $20 \%$, which was mainly originated from the determination of the irradiance distribution used in the experiment, i.e., beam waist, pulse width and laser power calibration.

With ultrashort pulses, it is important to account for a possible increase of pulse duration due to group velocity dispersion (GVD) in the samples. Pulse broadening may alter the peak irradiance and hence the magnitude of the nonlinear phase shift. We evaluate the dispersion distance, $L_{\mathrm{D}}$, defined as the length over which a transform-limited Gaussian pulse broadens by a factor $\sqrt{2}$ due to second-order dispersion [10]:

$L_{\mathrm{D}}=\frac{\Delta \tau^{2}}{4 \ln 2}\left(\frac{\lambda^{3}}{2 \pi c^{2}} \frac{\mathrm{d}^{2} n}{\mathrm{~d} \lambda^{2}}\right)^{-1}$,

where $\Delta \tau$ is the FWHM input pulse duration. The pulse broadens to an FWHM duration $\Delta \tau^{\prime}$ after propagating a distance $z^{\prime}$ inside the crystal,

$\Delta \tau^{\prime}=\Delta \tau \sqrt{1+\left(z^{\prime} / L_{\mathrm{D}}\right)^{2}}$.
The extent of pulse broadening is governed by $L_{\mathrm{D}}$. For a given pulse duration, short sample length makes the pulse broaden less. When the sample length is over $L_{\mathrm{D}}$, pulse broadening becomes important for pulse evolution. Using Eq. (1), the dispersion distances for the samples are calculated as listed in Table 1. The calculations are based on the appropriate Sellmeier equations for each crystal, corresponding to the laser polarization and crystal orientation used in our measurements. From Table 1, one can see that the dispersion distance for $150 \mathrm{fs}$ pulses at $780 \mathrm{~nm}$ ranges from $7.1 \mathrm{~mm}$ in LN:Mg to $107 \mathrm{~mm}$ in LBO. Hence, with the 2- and 5-mm samples, pulse broadening due to GVD is insignificant under our experimental conditions.

\section{Results and discussion}

The measured open-aperture Z-scans for all the samples exhibit that the Z-scan traces with the input irradiance up to $20 \mathrm{GW} / \mathrm{cm}^{2}$ are nearly flat, indicating that two-photon absorption does not occur in these NLO crystals. This is anticipated because the band-gap energies of these crystals are greater than the energy of two $780 \mathrm{~nm}$ photons. As examples, Figs. 1 and 2 show typical closed-aperture Z-scans on LN:Mg and KTP obtained with linear polarized $780 \mathrm{~nm}, 150 \mathrm{fs}$ pulses at different incident irradiances. Note that all the input irradiances reported here are the peak irradiances at the focus within the samples.

The closed-aperture Z-scans are sensitive to both the nonlinear absorption and nonlinear refraction. We assume that the total refractive index $n=n_{0}+n_{2} I$, where $I$ is the irradiance of the laser beam within the sample. As indicated in the preceding section, our samples with $2 \mathrm{~mm}$ and $5 \mathrm{~mm}$ thickness did not satisfy the "thin sample" condition. Hence, the standard Z-scan theory [4] with the "thin sample" limit is not applicable to our case. Hermann and McDuff [11] have derived an analytic expression for the Z-scan of a thick sample $\left(L>n_{0} z_{\mathrm{R}}\right)$, corrected to the first order in irradiance, for a pinhole aperture in the far field. In a slightly different form, the expression for the normalized transmittance $T$, may be written [12]: 


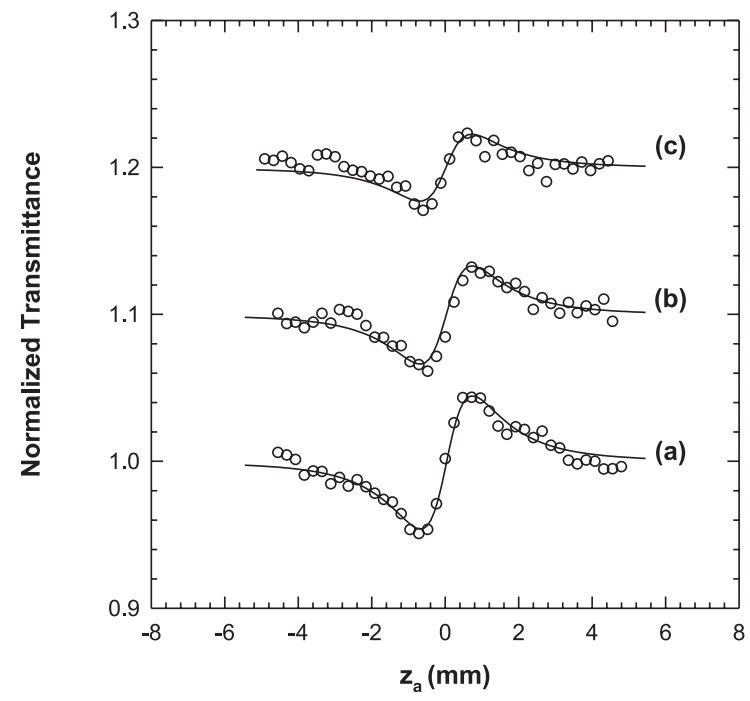

Fig. 1. Closed-aperture Z-scan measurements on the 2-mm thick $z$-cut LN:Mg crystal with the laser polarization along the [1 100 ] direction of the crystal. The open circles are measured at the input irradiances: (a) 10.0, (b) 7.9 , and (c) $5.3 \mathrm{GW} / \mathrm{cm}^{2}$. The solid curves are the theoretical fits described in the text. The data in (b), and (c) are vertically shifted by 0.1 and 0.2 , respectively, for presentation.

$$
\begin{aligned}
T= & 1-\frac{1}{4}\left\{\Delta \Phi_{0 \mathrm{R}} \ln \left(\frac{1+v_{a}^{2}}{9+v_{a}^{2}} \frac{9+v_{b}^{2}}{1+v_{b}^{2}}\right)\right. \\
& +Q_{0 \mathrm{R}}\left[\tan ^{-1}\left(v_{a}\right)+\tan ^{-1}\left(v_{a} / 3\right)\right. \\
& \left.\left.-\tan ^{-1}\left(v_{b}\right)-\tan ^{-1}\left(v_{b} / 3\right)\right]\right\},
\end{aligned}
$$

where

$v_{i}=-\frac{1}{z_{\mathrm{R}}}\left(z_{i}+\frac{z_{\mathrm{R}}^{2}+z_{i}^{2}}{z_{1}^{\prime}-z_{i}}\right), \quad$ for $i=a, b$

$z_{a}$ is the distance from the position of the beam waist to the entrance face of the sample, $z_{b}=z_{a}+L / n_{0}$, and $z_{1}^{\prime}=z_{1}-L\left(1-1 / n_{0}\right)$ with $z_{1}$, the distance from the position of the beam waist to the pinhole in front of the detector. The nonlinear phase-shift and absorption parameters are $\Delta \Phi_{0 \mathrm{R}}=k n_{2} I_{0} n_{0} z_{\mathrm{R}}$, and $Q_{0 \mathrm{R}}=\beta I_{0} n_{0} z_{\mathrm{R}}$, respectively. The accuracy of the deduced expression (3) has been experimentally verified by Chapple et al. [13]. By applying Eq. (3), we calculate $T$ as a function of the $z_{a}$ position of the sample using $n_{2}$ as a free parameter, in which the nonlinear absorption

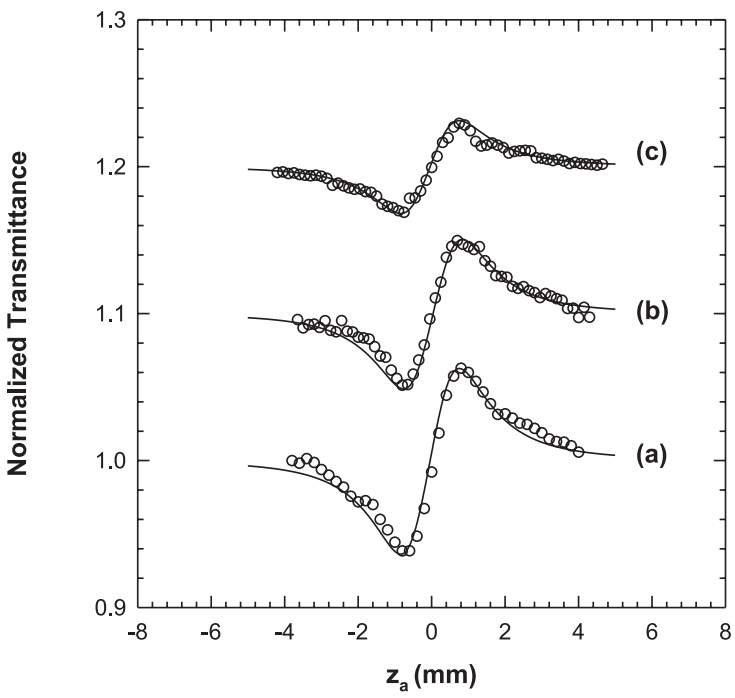

Fig. 2. Closed-aperture Z-scan measurements on the 2-mm thick $z$-cut KTP crystal with the laser polarization along the [1 $\left.\begin{array}{ll}0 & 0\end{array}\right]$ direction of the crystal. The open circles are measured at the input irradiances (a) 21.4 , (b) 17.2 , and (c) $10.7 \mathrm{GW} / \mathrm{cm}^{2}$. The solid curves are the theoretical fits described in the text. The data in (b), and (c) are vertically shifted by 0.1 and 0.2 , respectively, for presentation.

coefficient is regarded as zero. The $n_{2}$ values can be extracted from the best fitting. The solid lines in Figs. 1 and 2 show the best fits to the experimental closed-aperture Z-scan traces at different input irradiances, which yield a range of $n_{2}$ values. We plot these $n_{2}$ values versus the input irradiances in Fig. 3, which show that, within our experimental error, the measured $n_{2}$ for $\mathrm{LN}: \mathrm{Mg}$ and KTP is independent of the input irradiance, indicating that the observed nonlinear refraction is of the Kerr nonlinearity. The averaged $n_{2}$ values for $\mathrm{LN}: \mathrm{Mg}$ and $\mathrm{KTP}$ are $2.0 \times 10^{-15}$ and $1.2 \times$ $10^{-15} \mathrm{~cm}^{2} / \mathrm{W}$, respectively.

Similar Z-scans and analytical procedures have been carried out for the other crystals with the laser beam propagating along the $z$-axis with the polarization in the $\left[\begin{array}{lll}1 & 0 & 0\end{array}\right]$ and $\left[\begin{array}{lll}0 & 1 & 0\end{array}\right]$ directions. The measured $n_{2}$ values are summarized in Table 1 . One can see that the measured $n_{2}$ values for these NLO crystals range from $10^{-16}$ to $10^{-15} \mathrm{~cm}^{2} / \mathrm{W}$, and LBO has the smallest $n_{2}$ value while the largest $n_{2}$ value is given by $\mathrm{LN}: \mathrm{Mg}$. In addition, for KTP, $\mathrm{BBO}$, and LBO samples, the nonlinear refraction 


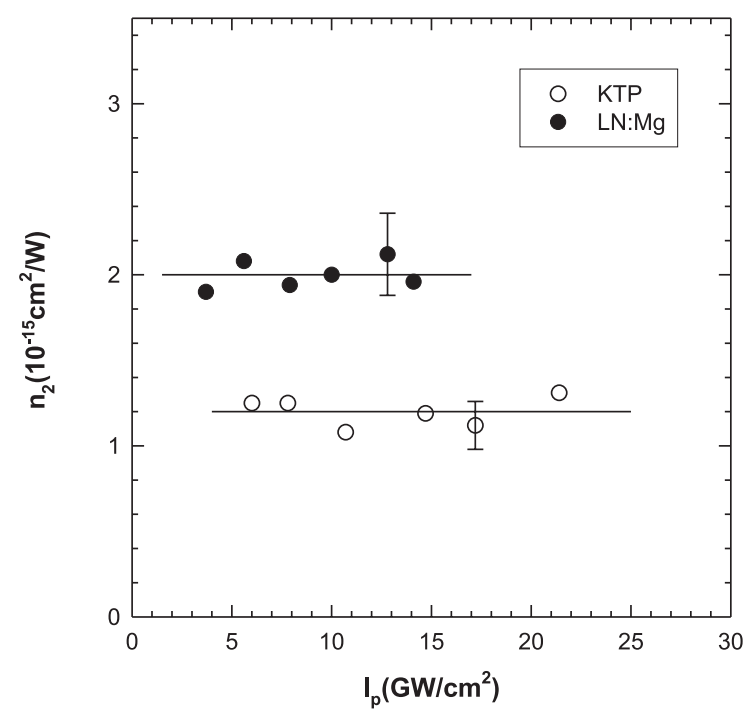

Fig. 3. Measured $n_{2}$ (open and closed circles) versus the input irradiance, $I_{\mathrm{p}}$, for LN:Mg and KTP. The solid lines are guides for the eyes.

varies with the beam polarization directions, indicating the anisotropy of the third-order nonlinearities in these crystals.

All the Z-scan measurements as reported above were conducted with $780 \mathrm{~nm}$ laser pulses at a repetition rate of $76 \mathrm{MHz}$. In order to estimate thermal contribution to the measured refractive nonlinearity, we also made similar Z-scan measurements on the samples with a lower repetition rate of $760 \mathrm{kHz}$, and no difference was found. This suggests that the thermal-lensing effect was weak under our experimental conditions, and the measured refractive nonlinearity is predominately electronic in origin. It might be due to the fact that the linear and nonlinear absorptions of the samples are insignificant at the excitation wavelength of $780 \mathrm{~nm}$.

Based on the two-band model with a simple direct bandgap, Sheike-Bahae et al. [14] have calculated the dispersion and band-gap scaling of the bound electronic Kerr nonlinearity, $n_{2}$, in solids. The nonlinear refractive index is given by

$n_{2}(\mathrm{esu})=K^{\prime} \frac{\sqrt{E}_{\mathrm{p}}}{n_{0} E_{\mathrm{g}}^{4}} G_{2}\left(\frac{\hbar \omega}{E_{\mathrm{g}}}\right)$,

where $K^{\prime}$ is a material-independent constant with a

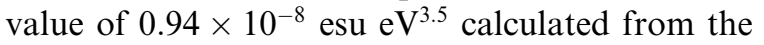

two-band approximation, $E_{\mathrm{p}}$ is the Kane momentum-related parameter with a value near $21 \mathrm{eV}$ for a large number of materials, and $G_{2}\left(\hbar \omega / E_{\mathrm{g}}\right)$ is a function only of the ratio of the photon energy to the energy gap of the material. By using Eq. (4), the theoretical $n_{2}$ values for the NLO crystals are calculated as listed in Table 1 . The theoretical $n_{2}$ values for $\mathrm{LBO}, \mathrm{BBO}$ and $\mathrm{Mg}: \mathrm{LN}$ are close to experimental results, whereas the theory overestimates $n_{2}$ for KTP and KTA. Eq. (4) shows that these crystals have different $n_{2}$ values, resulting from different band-gap energies. Due to the wider band gaps, the calculated $n_{2}$ values in BBO and LBO should be one order of magnitude smaller than those in LN:Mg, KTA and KTP, which is consistent with our measurements. In addition, it should also be noted that this theory does not explain the difference of $n_{2}$ values for different laser beam polarization and propagation, which needs further modification.

\section{Conclusion}

We have carried out an investigation of thirdorder optical nonlinearity in several important NLO crystals using the Z-scan technique with 780 $\mathrm{nm}$ femtosecond laser pulses. The nonlinear refractive index $n_{2}$ in these crystals has been determined to be in the range from $10^{-16}$ to $10^{-15} \mathrm{~cm}^{2} /$ W. No two-photon absorption has been observed. It is found that the measured $n_{2}$ values in BBO and LBO are one order of magnitude smaller than those of $\mathrm{LN}: \mathrm{Mg}$, KTA and KTP, which is consistent with the theoretical prediction based on the two-band model for the bound electronic Kerr nonlinearity. In addition, the experimental results show that nonlinear refraction in KTP, BBO and LBO is dependent of the polarization of the laser beam.

\section{References}

[1] T.J. Driscoll, G.M. Gale, F. Hache, Opt. Commun. 110 (1994) 638.

[2] J.M. Dudley, D.T. Reid, M. Ebrahimzadeh, W. Sibbett, Opt. Commun. 104 (1994) 419. 
[3] E.S. Wachman, D.C. Edelstein, C.L. Tang, Opt. Lett. 15 (1990) 136.

[4] M. Sheik-Bahae, A.A. Said, T.H. Wei, D.J. Hagan, E.W. Van Stryland, IEEE J. Quantum Electron. 26 (1990) 760.

[5] R. DeSalvo, M. Sheik-Bahae, A.A. Said, D.J. Hagan, E.W. Van Stryland, Opt. Lett. 18 (1993) 194.

[6] R. DeSalvo, A.A. Said, D.J. Hagan, E.W. Van Stryland, M. Sheik-Bahae, IEEE J. Quantum Electron. 32 (1996) 1324.

[7] H.P. Li, F. Zhou, X.J. Zhang, W. Ji, Opt. Commun. 144 (1997) 76.

[8] T.D. Krauss, F.W. Wise, Appl. Phys. Lett. 65 (1994) 1739.
[9] R. DeSalvo, D.J. Hagan, M. Sheik-Bahae, G. Stegemen, E.W. Van Stryland, H. Vanherzeele, Opt. Lett. 17 (1992) 28.

[10] G.P. Agrawal, Nonlinear Fiber Optics, Academic Press, New York, 1989.

[11] J.A. Hermann, R.G. McDuff, J. Opt. Soc. Am. B 10 (1993) 2056.

[12] P.B. Chapple, J. Staromlynska, J.A. Hermann, T.J. Mckay, R.G. McDuff, J. Nonlinear Opt. Phys. Mater. 6 (1997) 251.

[13] P.B. Chapple, J. Staromlynska, R.G. McDuff, J. Opt. Soc. Am. B 11 (1994) 975.

[14] M. Sheik-Bahae, D. Hutchings, D.J. Hagan, E.W. Van Stryland, IEEE J. Quantum Electron. 27 (1991) 1296. 